\title{
Problematizando los abordajes de la seguridad híbrida en América Latina
}

\section{Rafael EnriQue Piñeros Ayala*}

\section{RESUMEN}

La gobernanza global es un enfoque que se incorpora con mayor interés en el estudio de las Relaciones internacionales. Su versatilidad y utilidad amplían el alcance conceptual, metodológico y práctico de la disciplina, lo cual permite investigar y explicar las interacciones y procesos en ocasiones poco claros que se producen entre actores de distinta naturaleza. Desde esta aproximación, la Gobernanza se ha erigido como una herramienta que amplía las explicaciones de los enfoques conceptuales y metodológicos tradicionalmente utilizados, en otras palabras, la gobernanza es un enfoque multidisciplinario y reflexivo que logra desvanecer fronteras interpuestas por los lentes teóricos convencionales y adicionalmente genera nuevas comprensiones entre lo global y lo local.

Con base en lo anterior, se ha abierto y estimulado la utilización de la gobernanza al estudio de los órdenes regionales, pues aquellos se encargan de gestionar la agenda internacional, variada y compleja, ubicándose en un punto medio entre dinámicas globales y afectaciones locales. En ese sentido, ha contribuido a fortalecer una comprensión holística de los patrones históricos de comportamiento entre los miembros que los conforman, otorgándole mayor profundidad, ampliación y reflexividad a los procesos y desafíos allí presentes, así como a las respuestas requeridas a disyuntivas estructurales y coyunturales que evolucionan o emergen.

En ese sentido, América Latina ha vivido en los últimos veinte años importantes transformaciones en la forma en la que se relacionan los estados y otros actores. La agenda de seguridad no ha sido ajena al fenómeno, por el contrario, el estudio de la gobernanza de seguridad se ha insertado como una categoría adicional de estudio. Así las cosas, la seguridad contemporánea emerge de una combinación entre estructuras políticas, sociales y culturales, que reflejan tendencias históricas, así como nuevos marcos de comprensión analítica, generando un espacio gris, híbrido y heterogéneo.

El objetivo central de este artículo es establecer aquellas variables teóricas y prácticas necesarias para el estudio de la gobernanza híbrida de seguridad en América Latina a lo largo del siglo XXI a partir de un enfoque metodológico analítico, ecléctico y situado que señale los elementos distintivos y al mismo tiempo disputados o enfrentados que le han dado forma a la región. Se señala entonces que, la gobernanza híbrida seguirá alterando las definiciones tanto de seguridad como de región, conforme se alteren las dinámicas de poder, los principios y valores que guían el comportamiento de los agentes involucrados y la eficacia que tengan las organizaciones regionales para la gestión de los desafíos presentes y futuros.

Para lo anterior, este escrito consta de tres partes. En la primera, se hará una revisión exhaustiva de las definiciones y alcance de la gobernanza global para así facilitar la comprensión sobre su utilidad teórica, revisando algunas críticas. Posteriormente se validará su beneficio para formular nuevos marcos de comprensión acerca de los órdenes regionales y las características que allí se forjan y, finalmente, se empleará para mostrar cómo ha consolidado una estructura heterogénea, disputada y conflictiva que no se ha terminado de completar o de desarrollar en América Latina.

\section{Palabras clave}

Gobernanza global;América Latina; seguridad híbrida; región, regionalismo.

\section{TitLE}

Challenging Hybrid Security Approaches in Latin America

\section{EXTENDED Abstract}

The Global Governance has been increasingly used in the study of International Relations which, thanks to its versatility and utility, has generated an expansion of its conceptual, methodological, and practical scope. Regional governance is a useful derivation because it makes it easier to understand, from a more precise perspective, how global issues with local repercussions are solved.

\section{DOI:}

https://doi.org// 0.15366/relacionesinternacionales2021.48.010

Formato de citación recomendado:

PIÑEROS AYALA, Rafael Enrique (202I). "Problematizando los abordajes de la seguridad híbrida en América Latina”, Relaciones Internacionales, $\mathrm{n}^{\circ}$ 48, Pp. 193-211. 
In this sense, the article shows that security governance in Latin America is characterized by enduring balance of power practices, geopolitics, and mistrust between different parties. At the same time there is coexistence between the historical intention to establish security communities, to find resolutions to controversies, and to solve disputes through institutional instruments, principles, and values that favor peaceful means. In other words, in the region we are in the presence of a hybrid security governance, with practices that evolve over time in accordance with three key factors: the behavior of States and other agents, the capacity of institutions to overcome structural and conjunctural challenges, and finally, the effects of changes in perceptions of organized crime and other ideological and political challenges.

Therefore, this article consists of three parts. In the first, the definitions and scope of global governance will be reviewed. Subsequently, its utility will be validated for the comprehension of regional orders and the characteristics that are forged there. Finally, it will be used to expose the hybrid characteristics in Latin America throughout the 2 Ist century.

Traditionally, since its inception as a scientific discipline, the explanations provided by International Relations, have been the subject of multifaceted debates. In fact, it was through these great debates, sometimes theoretical and in other cases methodological, that the discipline grew in terms of academic and research production, as well as in the political scope for decision-making. This led to the creation of a true field of study, which took as its initial object of study the area of war and peace between nations, before later moving on to cooperation, the environment, and other issues of the contemporary international agenda.

Likewise, governance has been an ideal vehicle to give greater scope and heterogeneity to explanations of processes and empirical events, ones which go beyond traditional theoretical approaches such as realism, institutional liberalism or constructivism.

Along the same lines, this progressive academic production has stimulated the specialization and concentration of governance in regional orders, since it allows a clearer reflection on the historical patterns of behavior among the members. This offers a more nuanced explanation of the challenges present, the responses required for structural or conjunctural issues, and the eventual emergence of distinctive elements between different groups.

Security governance has mainly focused on four challenges to solve. First, the expansion of the research agenda to specific contexts; second, the greater attention that academic production should give to the relationship between intergovernmental organizations and other non-state actors in defined spaces; third, the predominance of explanations on security in the EuroAtlantic region and, finally, the need to further link the security governance agenda to debates on region and regionalism.

In the same way, the construction and specific behaviors developed in the regions cannot be detached from global dynamics. In this sense, regardless of the geographical character of a region such as Europe or South America, these are mainly political factors, as Hurrell well recognizes, that regional dynamics are a historical social construction, which has been politically contested and criticized. with the intention of recognizing patterns of continuity and transformation, which facilitate the identification of dynamics that particularize and distinguish between them.

In this sense, Latin America has not neglected the use of governance, as a concept and as a valid analysis methodology to understand the distinctive characteristics that have made up a particular set of elements that, on the one hand, endure over time and, on the other hand, have recently changed.When referring to security, the opposing points of view show the emergence and incipient development of a heterogeneous, incomplete, and controversial governance. The main objective of this article, based on a hybrid and eclectic approach, will be to understand the evolution of the concept of governance and establish the characteristics of security for Latin America.

Thus, in matters of security governance, this article highlights the hybrid character from two lines of argument; on the one hand, there are defenders of a vision based on geopolitics, the balance of power and the latent tension between states; and on the other, there is one more oriented to analyze security characteristics from a constructivist, associative and cooperative perspective. Indeed, Adler and Greve presented an interesting initial reflection on the subject, in the sense that both are necessary for the holistic understanding of the movements, not always ascending, that states carry to equip themselves with instruments and mechanisms that enable them. They allow you to live together in a more harmonious and peaceful way.

First, some authors situate regional governance in mainly realistic terms, in which the dynamics of balance of power between states continue to prevail two hundred years after independence. They underline, for example, realities of ungovernability, while a series of specific variables, among which at least five stand out: first, the old geopolitical tensions between states continue to determine situations of conflict and mistrust; secondly, the competencies of individual and personal leaders in provision of common goods and dispute resolution stand out. Third, regional disputes have continued to be fueled both by internal competition - that is, between the states of the region - and, at the same time, by permissiveness in the face of the influence of external powers; Fourth, the importance of the quality of democracy for the countries of the region, which facilitates internal governance processes and strengthening the rule of law and, finally, the link between security and the economy in terms of economic development, that is, the effect of economic independence and economic autonomy in strengthening state security.

Second, there is the most interpretive line of reflection on reality, highlighting that beyond the instruments of power and geopolitical, political processes and new ideological realignments must be understood, in which the following variables can be highlighted: first, the internal factors that condition or reinforce the international action of the States; second, the regional redefinition based on the creation of alternative multilateral forums, beyond the OAS, such as CELAC, UNASUR or ALBA, in which there are also components not only of trade and integration, but also of security and, finally, the little regional cooperation in security with low institutional, political and budgetary density.

\section{KEYWORDS}

Global governance; Latin America; hybrid security; region; regionalism. 


\section{ntroducción}

La Gobernanza global ha incrementado su importancia y relevancia como categoría explicativa, metodología de investigación y vehículo de aproximación a las Relaciones Internacionales. Así, es un medio y un fin en sí misma que refuerza tanto la multidisciplinariedad de lo internacional, como los cambios acaecidos dentro de la disciplina, la adaptación a situaciones cambiantes de entornos regionales y locales y, por último, una forma específica de entender la agenda internacional y las vías para solucionar problemas comunes.

Tradicionalmente, las explicaciones propuestas por la disciplina han sido objeto de profundos debates. Gracias a la identificación de grandes disputas, en ocasiones teóricas y en otras metodológicas, se puede afirmar que la producción académica e investigativa fue creciendo, así como su alcance político y su utilidad para la toma de decisiones. La consolidación de un verdadero campo de estudio pasó, entonces, por la necesaria identificación de un objeto de estudio, inicialmente sobre la guerra y la paz entre naciones y, posteriormente, desplegado a otras cuestiones como la cooperación, el medio ambiente y otros temas de la agenda internacional contemporánea (Lozano Vázquez, 2019, pp. 9-27).

Lo anterior ha producido al menos dos tendencias a resaltar. Por un lado, una disciplina con estructura líquida (Del Arenal, 2019, pp. 45-83) en la cual, continua evidenciándose una constante autocrítica y autorreflexión sobre las bases conceptuales, metodológicas, teóricas y normativas sobre las cuales se construye el conocimiento $y$, por otro lado, un creciente número de voces que convocan la inclusión, al menos, de ciertas variables dejadas de lado: La economía política internacional, la sociología histórica, las demandas y visiones de los pueblos autóctonos o sometidos al poder colonial y, por último, los aportes teóricos y prácticos de las mujeres tradicionalmente excluidas (Sanahuja, 2019, pp. 18I-208).

En efecto, el debate permanente incentiva renovar la manera en la que se estudian los fenómenos internacionales a partir de dos reflexiones contemporáneas en las ciencias sociales. En primer término, un giro post positivista e interpretativo que estimula la contestación sobre qué se estudia y cómo se explica y, en segundo término, la necesidad de incluir otras realidades diferentes a la ya clásica visión euroatlántica, señalando cómo en otros contornos regionales o culturales se afectan también las dinámicas de poder, de conocimiento y de recursos disponibles lo que facilita ampliar la comprensión internacional de la realidad (Tickner y Wæver, 2009, pp. I-32).

Así las cosas, es pertinente señalar que la disciplina afronta hoy un triple reto: por un lado, la inclusión de saberes provenientes de otras disciplinas; adicionalmente, la explicación de una agenda más amplia y con características diferenciadas y, finalmente, el esclarecimiento más diáfano de la interacción entre actores diversos y distintos al estado, con lo cual se evidencia el incremento de la influencia de individuos, empresas y ONG, que disminuye la capacidad de control y gestión estatal (Caporaso y Madeira, 2012).

La gobernanza global, entonces, se entiende como "la interacción en red entre agentes públicos y privados, tanto de carácter horizontal como vertical, que no solamente son estatales, sino que incluyen actores formales e informales de la sociedad civil” (Triandafyllidou, 20I7, p. 4). Con la anterior se subraya que, gobernanza implica cierta heterogeneidad y horizontalidad en 
los aportes provenientes de diversos agentes. En la década de los noventa, una de las primeras aproximaciones sobre la materia en cuestión, fue formulada por James Rosenau, quien recalcaba que "la autoridad sufre reubicaciones constantemente, tanto hacía afuera, es decir, entidades supranacionales, como internamente, a favor de grupos subnacionales, lo que refleja la evolución y eventualmente la ausencia de un gobierno formal (I992, p. 2).

Rosenau también planteó uno de los elementos distintivos de la gobernanza, en el sentido que esta desafía los mecanismos tradicionales de autoridad, proponiendo ejes alternativos en los que puede estar o no incluida la jurisdicción estatal. Aunque hoy parece normal que actores privados se organicen por sí mismos, tal como lo hacen las empresas alrededor de foros como el Pacto Global, en los cuales acogen voluntariamente principios generales que rigen su comportamiento corporativo, en los noventa resultaba revolucionario y herético plantear alguna forma de exclusión pública.

Se suele entender que la gobernanza se utiliza exclusivamente para comprender y estudiar marcos de relacionamiento o interacción entre actores, agendas, procesos e instituciones en ámbitos lejanos de decisión. Por el contrario, este trabajo se concentra en señalar que la gobernanza aplica de manera más efectiva cuando estudia contextos particulares o específicos de relacionamiento. En otras palabras, la agenda contemporánea presenta fenómenos que, como los expuestos previamente, son de naturaleza transnacional pero las prácticas y los procesos están atados a la historia y los principios y valores con carácter contextual (Triandafyllidou, 2017, pp. 3-24).

Así las cosas, la gobernanza puede tener un carácter diferente según el lugar desde el cual se observe. Posiciones de poder, zonas marginadas por al globalización económica o grupos políticos reaccionarios tendrán una perspectiva diferente sobre el mismo proceso (Tickner, 2003, pp. 325-350). Lo anterior nos presenta, al menos dos provocaciones:

Por un lado, las regiones adquieren un marco de referencia idóneo para la gestión de la agenda contemporánea a partir de la interacción entre estados y otros actores y las capacidades efectivas de las instituciones u organizaciones presentes (Herz, 20I4, pp. 236-250). Aquellas son un eje de articulación entre sucesos globales y efectos locales, entre marcos de acción distantes y resultados comunitarios contestatarios. Por el otro, el ascenso de la teoría crítica conlleva un relativo abandono de los marcos tradicionales para favorecer miradas reflexivas sobre las dinámicas de poder, los espacios regionales geográficos o políticos y la inclusión de actores diversos con epistemologías de estudio que descentralicen el conocimiento (Muhr, 20 I9, pp. 95-II I). Esta situación estimula la construcción de enfoques basados en el pragmatismo metodológico para incluir contextos particulares, que impulsan marcos eclécticos para el estudio y la práctica de las relaciones internacionales (Katzenstein y Sil, 2008, pp. 109-130).

Precisamente, América Latina —-más diversa y heterogénea — no ha sido ajena a la utilización de la gobernanza como categoría de estudio. De hecho, esta ha adquirido una forma multidimensional que envuelve asuntos económicos, culturales, militares, políticos y sociales (Herz, 20l4, p. 238), sin que con ello se desconozca la riqueza histórica sobre el desarrollo del multilateralismo, con sus ciclos de avances, retroceso y ocasional estancamiento a lo largo del siglo XX (Dabène, 20I2, pp. 4I-64). 
Justamente, al utilizar la gobernanza en los asuntos de seguridad en marcos regionales, se espera romper los lentes tradicionales para esclarecer nuevas relaciones de poder ocultas entre actores e instituciones, a partir de "observar e interpretar cambios en las dinámicas de la región y la subregión, señalando los límites geográficos o políticos que condicionan la participación de actores externos y cómo afectan, al menos, ciertos hechos aislados e independiente en el tipo de relación que llevan a cabo los actores" (Breslin y Croft, 2012, p. 5). En otras palabras, la gobernanza regional de seguridad otorga explicaciones — nuevas o diferentes - a los vínculos de seguridad entre estados y otros actores, valorando las relaciones políticas sociales o culturales, analizando la afectación de las dinámicas del crimen transnacional y otras prácticas securitizadas.

Así, este análisis se encamina a resolver la pregunta ¿cómo se constituye la gobernanza de seguridad en América Latina?, para lo cual se parte de que aquella es un reflejo tanto de prácticas históricas de comportamiento - especialmente entre los estados-como de nuevas conductas producidas por la intervención de otros agentes, generando un espacio híbrido de confrontación entre visiones opuestas que, por un lado, favorecen elementos estratégicos y de equilibrio de poder $y$, por el otro, factores institucionales de solución pacífica de controversias. En efecto, la gobernanza híbrida de seguridad regional se encuentra permeada por elementos de diálogo, ruptura y disputa, acerca del predominio de lo estructural y lo coyuntural, que altera las relaciones de poder y la visión de los agentes respecto a la afectación del crimen transnacional organizado y la capacidad institucional para superar desafíos al estado de derecho y la gobernabilidad.

Este artículo presenta así tres secciones: en un primer apartado se hará una reflexión sobre el alcance y significado de la gobernanza global, posteriormente se elaborará una correlación entre las dinámicas regionales y de seguridad para decantar, por último, los elementos distintivos que se mantienen o proyectan en América Latina.

\section{La Gobernanza global como aproximación a los estudios internacionales}

Este apartado desarrolla los amplios usos y aportes que para las Relaciones Internacionales tiene la gobernanza, señalando como erróneo abrogarse un enfoque específico como el más importante. Este concepto genera múltiples visiones no excluyentes entre sí, razón por la cual, corresponde explorar algunos de los significados más utilizados, manteniendo una perspectiva abierta a la inclusión de aportes que provienen de otras disciplinas.

La gobernanza, entendida como concepto, facilita ordenar y estructurar la observación con la intención de generalizar proposiciones encaminadas a percibir con lentes más allá de los teóricos convencionales (Dingwerth y Pattberg, 2006, p. 187), lo que permite verla tanto como instrumento propicio para la reflexión de lo internacional, al tiempo que como propuesta de investigación y desarrollo dentro de la disciplina.

De lo anterior se desprenden, al menos, tres implicaciones: en primer lugar, la gobernanza es una herramienta efectiva para observar una realidad ampliada, diversa y compleja; en segundo lugar, se constituye como un proyecto político que identifica ejes temáticos sobre los cuales as- 
pira a disminuir el papel y la influencia estatal en la solución de problemas y, finalmente, implica una actitud normativa que favorece repensar los acuerdos vigentes, con la vocación de reflejar de manera más clara los contornos históricos, sociales y culturales (Luckhurst, 2019, Pp. 176 - 194).

Al referirse a la gobernanza como categoría de estudio, inicialmente, puede ser interpretada a partir de la evolución del término. En el siglo XV hay vestigios de su utilización para referirse a cierta forma de gobierno y gestión (Marín-Aranguren y Trejos-Mateus, 2020, pp. 69-I I6). Asimismo el multilateralismo incipiente creado a finales del siglo XIX y principios del $X X$ tiene elementos que han perdurado hasta nuestros días, en referencia a la inclusión de mecanismos de gestión y control para solucionar problemas comunes. En esencia, los instrumentos para regular la guerra, la aviación comercial, el transporte marítimo, entre otros, son ejemplo en los cuales el creciente intercambio, requirió instrumentos creativos de organización, gestión y regulación (Murphy, 20I4, pp. 23-24).

Un segundo período se refiere al multilateralismo establecido después de la segunda guerra mundial con la creación del sistema de Naciones Unidas, las instituciones financieras para la reconstrucción de los países —Banco Mundial y Fondo Monetario Internacional, principalmente- y la administración del comercio de bienes y servicios alrededor del GATT, hoy OMC. Esa construcción de regímenes internacionales no sólo favoreció el desarrollo y la ampliación del multilateralismo, sino que, con el tiempo estimuló vías distintas a las exclusivamente intergubernamentales para administrar y gestionar los problemas que resultaban de la mayor interacción e interdependencia internacional (Murphy, 2014, pp. 25-35).

Un tercer período de creciente importancia fueron los años ochenta y noventa. Los efectos de la globalización comercial, social y política, la erosión de la capacidad del estado para dar respuesta adecuada a los problemas comunes e interdependientes en seguridad, medio ambiente o migraciones $y$, al mismo tiempo, una actividad más dinámica, propositiva pero crítica de actores de la sociedad civil, impactó en que la gobernanza fuera vista desde dos ópticas: por un lado, como producto o necesidad de los mercados y de las estructuras de poder imperantes y, por el otro, como una faceta de reacción y crítica frente a las dinámicas de mayor difusión de poder (Barnett y Duvall, 2005, pp. I-32). En cierto modo, las ventajas incuestionables de un orden liberal democrático y de libre mercado consolidado después de la caída de la Unión Soviética no eliminó las críticas sobre la evolución de la disciplina, los efectos de la globalización o la gestión de la gobernanza.

Con base a lo anterior, la evolución de los fenómenos internacionales, por un lado, y los procesos de globalización crecientes pero críticos, por el otro, son evidencia clara que hoy se requiere más y no menos gobernanza (Caporaso y Madeira, 2012). Precisamente, la toma de decisiones hecha por los estados y aquellas que se toman en otras instancias de decisión —como las Organizaciones Internacionales - se ha volcado en incrementar la participación de otros jugadores y también a discutir sobre una agenda más amplia, diversa y heterogénea.

Como lo reflejan algunos autores, la gobernanza se define como un "punto de encuentro complejo entre instituciones, mecanismos, procesos y relaciones formales e informales, que vinculan a estados, mercados, ciudadanos y organizaciones, cuyos intereses colectivos son articulados mediante derechos y participaciones previamente establecidas" (Thakur y Van Langenhove, 2006, p. 235). 
Sobre lo anterior, es pertinente señalar que el multilateralismo no es gobernanza y que está última no es solo multilateralismo. La primera, en un sentido clásico, se refiere a la necesidad de que diferentes lados en igualdad de condiciones acuerden trabajar sobre asuntos en común y construir consensos sobre la mejor manera de abordar un tema (Ruggie, I 993, pp. 3-5I) y, como se ha abordado, la gobernanza se refiere a la institucionalidad y su agencia, a niveles de actividad humana que involucran ejercicios participativos, formas de organización individuales y colectivas (Marín-Aranguren y Trejos-Mateus, 2020, pp. 69-1 I6), así como acuerdos y decisiones sobre agendas diversas, entre otras, comercio internacional, salud, seguridad internacional, participación ciudadana.

Lo que se quiere subrayar en este punto, se refiere a que el estudio del multilateralismo y de las organizaciones internacionales puede recibir un impulso positivo con la utilización conceptual y metodológica de la gobernanza, es decir, esta última actúa como medio alternativo para el desarrollo de la primera, alrededor de al menos tres derivaciones conceptuales: buena gobernanza, gobernanza multinivel y gobernanza como concepto.

La primera recuerda que son aquellos principios y códigos de comportamiento establecidos para hacer el gobierno más justo, sensible a la administración de justicia y los derechos humanos, la transparencia y la rendición de cuentas (Kjær y Kinnerup, 2002, Pp. I- 19); la segunda se refiere a la toma de decisiones efectuada en diferentes niveles de decisión, que puede ir de lo local a lo supranacional, reflejando una dispersión de la autoridad en diferentes unidades de gestión (Fabrinni, 2020; Kirchner, 2014) y, finalmente, la gobernanza como concepto, tal como lo definió la Comisión sobre Gobernanza Global auspiciada por Naciones Unidas:

"Es un proceso amplio, dinámico y complejo de toma de decisiones interactiva, que cambia y se adapta a circunstancias cambiantes, dentro de instituciones formales y no formales, que empodera regímenes internacionales para mejorar el cumplimiento y lograr el mejor interés para individuos y estados" (UN, 1995).

La gobernanza, entonces, empodera y refuerza construir una autoridad epistémica, que en ocasiones adopta principios corporativos para alcanzar mejores resultados, dentro de marcos institucionalizados o no, que disminuyen el papel del estado y aumentan el nivel de participación de otros actores y foros de decisión (Weiss y Wilkinson, 2014; Jönsson, 2018, pp. 3-19). Así, se pretende ir más allá del multilateralismo convencional, en el sentido que refuerza la noción de la interconexión de lo político con la toma de decisiones, estimula construir decisiones colectivas para reforzar acuerdos vinculantes sin dejar de lado la competencia entre actores diversos.

A pesar de su importante y creciente aceptación, como es natural, la gobernanza no está exenta de críticas sobre su valor y utilidad para mejorar la comprensión de los fenómenos que estudia.Varias líneas se han consolidado sobre el mismo: en primer lugar, la transposición con el multilateralismo y la reproducción de prácticas de aquel, como la parálisis en la toma de decisiones, la debilidad de instrumentos que refuercen y hagan cumplir lo pactado (Jönsson, 2018) y, adicionalmente, la heterogeneidad y fragmentación de la comprensión y alcance de sus propósitos 
son considerados una penetrante debilidad en la medida que no se logra obtener el beneficio que, desde el discurso, pretende alcanzar (Weiss y Wilkinson, 2014, p. 3).

Una segunda línea podría ser la desarrollada por Zürn, en el sentido que la gobernanza ha crecido como un cuerpo normativo particular, una especie de autoridad que desafía la legitimidad y capacidad del estado, ocasionando una lucha constante por la legitimidad en su accionar. De ahí que se observe con suspicacia su utilización en temas como la seguridad internacional, el uso de la fuerza o la capacidad de instituciones financieras internacionales para determinar planes y proyectos económicos de gran envergadura, acrecentando la distancia entre ciudadanía y toma de decisiones descentralizada en gobernanza (Zürn, 20I8).

Acerca de la posible difusión de autoridad, las observaciones son mixtas o divididas. Por un lado, la difusión y ampliación del multilateralismo y la gobernanza han contribuido a la toma de decisiones que favorezca al ciudadano, empoderándolo para encontrarle solución a sus problemas (Held, 20l4). Por el otro, esa difusión creada por la eliminación de las fronteras entre lo nacional e internacional, la mayor capacidad presupuestal, técnica y de influencia de actores distintos al estado, así como los cambios en la manera que se hacen las leyes, genera espacios policéntricos y complejos en los que se genera tanto expectativa como rechazo, crítica y en ocasiones desinterés por parte de los estados (Held y Hale, 20I I), que en últimas toman las decisiones.

\section{La gobernanza en seguridad desde una perspectiva regional}

El propósito de este epígrafe es señalar la relación y los mecanismos de interacción entre gobernanza, seguridad y espacios regionales. Como se demostró previamente, la utilización de la gobernanza tiene diversas ventajas, incluso en espacios regionales y en diversos temas de la agenda internacional. Acá se reforzará la noción bajo la cual el estado nunca logró una plena seguridad, razón por la cual, se han instaurado no sólo instituciones multilaterales y procesos de integración, sino también una ampliación de actores que contribuyen a mejorar las condiciones de seguridad para el estado y los ciudadanos (Triandafyllidou, 2017, pp. 3-24).

Alrededor del concepto de seguridad, los debates sobre la ampliación del significado, el alcance del concepto y los métodos empleados para su estudio, comenzaron a ser trabajados y modificados poco antes del final de la guerra fría, con lo cual, los años posteriores fueron fructíferos en brindar diversas explicaciones sobre nuevos ejes temáticos, la inclusión de nuevos actores, la reflexión sobre el objeto y las dinámicas específicas de estudio (Adler y Barnett, 1998; Buzan, Waever, y Wilde, 1998; Buzan y Hansen, 2009; Frasson Quenoz y Piñeros Ayala, 2014).

Por lo cual, la seguridad se convierte en una más de las agendas abordadas por la gobernanza, materializando lo mencionado en la sección anterior, pues se busca "ejercer algún tipo de coordinación entre agentes sociales en los que se construyen imágenes, interpretaciones de la realidad, escenarios de solución de controversias en distintas áreas de la actividad humana" (Domínguez y Velázquez Flores, 2018, p. 4).

Inicialmente, los aportes de Ceccorulli y Lucarelli (20I4), caracterizan a la gobernanza en 
seguridad a partir de cuatro desafíos por resolver. En primer término, la necesaria ampliación de la agenda de investigación a contextos particulares; en segundo lugar, desarrollar de manera más clara la relación e interacción entre las organizaciones intergubernamentales y actores no estatales; en tercer lugar, superar el predominio de las explicaciones que sobre seguridad euroatlántica existentes y, por último, la necesidad de vincular más la agenda de gobernanza en seguridad a los debates sobre región y regionalismo (pp. 4-6). En otras palabras, hay que adaptar los estudios de gobernanza a regiones o zonas poco exploradas previamente que enriquezcan, como se señaló en la sección anterior, las nociones diferenciadas sobre seguridad y región.

Sobre este último aspecto, las discusiones sobre región y regionalismo adquieren en cada entorno geográfico o político una serie de atributos y características que las distinguen de los demás. En términos de seguridad, el final de la Guerra Fría abrió un espacio para que aquellas tomaran un lugar más protagónico en términos de desarrollar principios, instituciones y comportamientos que les permitieran encontrar soluciones a los problemas derivados de enemistades entre estados, dinámicas del crimen organizado, desafíos a la democracia o al estado de derecho, entre otros. En efecto, lo regional adquiere un protagonismo inusitado, como "mediadoras y facilitadoras en torno a la construcción de medidas de confianza mutua, mantenimiento de la paz, reforzamiento del estado de derecho y funciones adicionales que la región misma decida que pueda y quiera emplear" (MacFarlen, 20 I4, p. 430).

Precisamente, los comportamientos específicos desarrollados en las regiones no pueden ser excluidos de la relación entre estas y las dinámicas globales. Independiente del carácter geográfico — que le da forma a una región como Europa o América del Sur- son esencialmente los factores políticos los que determinan el tipo de relacionamiento entre los actores intervinientes, la influencia del poder constituido y contestado a través del tiempo y definen también las variables que señalan la importancia de una región (Flemes y Nolte, 20 I 0; MacFarlen, 20I4). Como lo afirma Hurrell, las dinámicas regionales son una construcción social histórica políticamente contestada y criticada, que se constituye con la intención de examinar patrones de continuidad y transformación que evidencien dinámicas que particularizan y distinguen unas de otras (2010, p. I8).

Así las cosas, las investigaciones sobre gobernanza en seguridad en ámbitos regionales, señalan una perspectiva propicia para abandonar prejuicios y modelos estáticos en el tiempo, ampliando la visión clásica de relacionamiento basado en asuntos de poder y de capacidades materiales, construcciones institucionales y organizacionales.

En una línea de investigación dirigida a estudiar el desempeño de las dinámicas regionales y de las organizaciones formales e informales, Kirchner y Domínguez (20I4) establecen una serie de variables, destacando las siguientes: en primer lugar, entender el alcance de las organizaciones regionales a partir de sus objetivos y mecanismos de actuación para conocer su efectiva utilidad; en segundo lugar, reconocer la importancia de instrumentos de coordinación y acción conjunta, tales como el desarrollo de medidas de confianza mutua, libros blancos o información propicia para disminuir tensiones; en tercer lugar, establecer los pasos destinados a implementar políticas comunes de seguridad o defensa, esto es, armonizar procesos, operaciones y definiciones comunes y, finalmente, alcanzar instrumentos de reforzamiento y supervisión de los acuerdos, es decir, saber qué sucede en caso que algún miembro no cumpla con lo pactado (pp.169-172). 
En contraposición, hay autores que enfatizan otras variables, tales como la influencia de potencias externas, la capacidad de las organizaciones estatales y los asuntos conferidos a estas, así como la incidencia de otros agentes —organizaciones de la sociedad civil-, con lo cual se consiguen crear espacios de aceptación o recurrente tensión con relación a la solución pacífica de controversias en una región (Breslin y Croft, 2012, p. 16).

Como sucede en cualquier proceso de construcción social y político, la gobernanza como método de aproximación a los fenómenos de seguridad, por ejemplo, implica comportamientos de negociación, cooperación, regulación y conflicto, que son dinámicos conforme se modifiquen condiciones estructurales o la coyuntura así lo demande. Es por esta razón que las interacciones antes descritas y cualquiera que pueda surgir, no necesariamente son neutrales, por el contrario, reflejan intereses, percepciones, temores, inseguridades de los actores intervinientes en los procesos de construcción. En ese sentido, resulta de utilidad mantener una perspectiva sobre gobernanza global y otro acerca de gobernanza regional que les permita diferenciarse, en tanto que la primera adquiere una dimensión transnacional amplia $y$, la segunda, una particular adaptación a contornos específicos, sectoriales y locales con algún tipo de diferenciación (Triandafyllidou, 2017, pp. 3-24)

Esa reflexión crítica y reflexiva, resulta conveniente porque extrae de manera más clara y situada aquellos vasos comunicantes entre dinámicas globales y locales, a partir de la identificación de singularidades propias de los comportamientos referentes al ejercicio de poder, los procesos de institucionalización o los principios y valores que desean alcanzar, respetar o practicar en el ejercicio diario de relacionamiento. En otras palabras, las regiones con características propias en seguridad, definidas como complejos (Frasson Quenoz y Piñeros Ayala, 2014, pp. I3-5I), comunidades (Adler y Barnett, 1998) o algún punto intermedio, aspiran a ejercer cierto poder, control o guiar los procesos particulares que se desarrollen en los contornos de actuación definidos por los actores intervinientes (Flemes y Nolte, 2010, pp. I- I5).

En efecto, resulta ineludible precisar las líneas de interacción entre comportamientos globales y particularidades regionales. Y es que estas últimas, al ser un eje articulador son partícipes de la gestión en ámbitos puntuales y acotados de la agenda internacional, destacando los siguientes comportamientos: en primer lugar, dinámicas de irrelevancia, cuando las regiones por su alcance limitado y su debilidad estructural son poco importantes y no influyen en lo global; en segundo lugar, encontramos comportamientos de conflicto y confrontación, en los cuales las dinámicas regionales o locales se convierten en un desafío para el orden, la seguridad o la estabilidad de espacios globales; en tercer termino, se emplazan las relaciones de cooperación y superposición, en el sentido que hay cierta simetría, previsibilidad o delegación en lo regional de elementos globales de toma de decisiones y, por último, las relaciones de harmonía en las cuales la dinámica de gobernanza regional complementa, armoniza o desarrolla instrumentos creados en foros globales con apropiación de factores culturales e idiosincráticos locales (Kacowicz, 20 I8, pp. 65-68).

La incidencia y relevancia entre las dinámicas globales y regionales están mediadas, adicionalmente, por una serie de comportamientos exhibidos por las potencias regionales. Una mayor actividad y presencia de aquellas en las dinámicas asociativas de la región, es decir, en la provisión de bienes públicos, el apoyo y desarrollo de los instrumentos de mercado o institucionales que 
contribuyan no sólo a su bienestar y crecimiento, sino también al de los demás países, son particularmente diferenciadoras en el sentido que fortalecen o debilitan la importancia estratégica de su entorno más próximo (Flemes y Nolte, 2010; Rocha Valencia y Morales, 201 I). Por lo anterior, el tipo de relacionamiento de las potencias regionales refuerza el carácter ecléctico de este artículo, en el sentido que dinamiza, expande y consolida, diferentes categorías de análisis para el estudio de las regiones y los comportamientos que allí se desenvuelven.

Por ello, para algunos autores, una adecuada gobernanza regional es el reflejo de la implementación de lo global con apropiaciones culturales particulares, en las que se facilita discernir con mayor precisión la capacidad efectiva que instituciones, procesos formales e informales tienen para la superación de desafíos prácticos, coyunturales o estructurales en espacios claramente definidos (Thakur y Van Langenhove, 2006, pp. 233-240). Es decir, la gobernanza regional traduce una forma de evaluación efectiva de los mecanismos a disposición para mejorar o mantener la seguridad y mejorar la confianza entre actores.

Para cerrar la discusión sobre los espacios de gobernanza regional en materia de seguridad, se torna adecuado el análisis propuesto por Murphy, en el sentido que el progreso de la gobernanza estará marcado por la evolución de las formas históricamente creadas y adaptadas a tiempos cambiantes, así como por los intercambios crecientes entre los mecanismos formales y los informales de relacionamiento (Murphy, 20I4, pp. 23-35).

Los debates sobre la transformación de la gobernanza regional estarán así inscritos, igualmente, por las discusiones y reflexiones sobre los cambios en la soberanía y legitimidad de los estados, las fuerzas cambiantes de la globalización y los desafíos a la representación y control democrático dentro y fuera del estado (Herz, 20 I4, pp. 239-242) que, como en el acápite anterior, permitió señalar ciertos elementos que deben ser atendidos con la intención de mejorar la efectividad de la gobernanza regional como categoría de estudio.

Finalmente, las definiciones y procesos llevados a cabo en lo regional no pueden ser entendidos como hechos concluidos, sino que, siempre abiertos al debate político-ideológico, la adaptación a amenazas coyunturales o la superación por otras formas más novedosas de asociación.

\section{Seguridad en América Latina: Gobernanza híbrida y en desarrollo}

Este acápite explica la gobernanza de seguridad en América Latina como un proceso híbrido, en desarrollo y permanentemente impugnado, construido a partir de una superposición de variables, características y patrones que se mantienen en el tiempo, así como otros adicionados por coyunturas particulares. Igualmente, la gobernanza de seguridad híbrida, ha sido construida y desarrollada principalmente por los estados, con alguna participación de instituciones regionales vigentes, así como por la influencia creciente de dinámicas del crimen transnacional organizado.

América Latina como región enfrenta un triple proceso de transformación con ramificaciones en seguridad: primero, una globalización económica en la que como región pierde competitividad respecto a Asia Pacífico; segundo, una competencia global por el poder entre Estados 
Unidos y China con ramificaciones locales, en el sentido de realineamientos políticos e ideológicos entre algunos países y, finalmente, un incremento en los intentos regionales de configurar instrumentos adecuados y autónomos que no necesariamente se consolidan y utilizan plenamente (Serbin, 2018, pp. 13-35)

Justamente, el siglo XXI ha sido testigo de esos cambios en los que, por ejemplo, se critica con vehemencia sin abandonar el multilateralismo establecido como una vía para la solución de controversias (Weiffen, 2017, pp. 173-198), en los que se desea tomar distancia parcial de Estados Unidos diversificando aliados políticos, económicos e ideológicos (Ellis, 20I6, pp. 29l-30l) y, en definitiva, anhela construir espacios renovados desde el Sur Global con la intención de transformar realidades locales (Sanahuja, 20I7, pp. I8I-208).

Esa heterogeneidad y complejidad política también ha tenido eco en términos de seguridad. Una de las primeras aproximaciones sobre elementos de convivencia híbrida fue expuesta por Adler y Greve (2019) para quienes la coexistencia de mecanismos de seguridad, instituciones y prácticas entre miembros de una región, reproducen comportamientos basados en el equilibrio de poder, la competencia geopolítica y la correlación de fuerza material, al tiempo que presentan otras formas de organización basadas en comunidades de seguridad, en las que se comparten principios, valores y la firme convicción de la resolución pacífica de controversias (pp. 59-84).

Más que ordenes excluyentes, los autores refuerzan la idea de la gobernanza híbrida a partir de elementos de convivencia y superposición de instituciones o prácticas que aseguren ordene estables (Adler y Greve, 2009, p. 65).

La región no ha sido ajena a esa situación. De hecho, en más de 200 años de vida republicana, han convivido ordenes de paz negativa, como las disputas históricas entre Argentina, Brasil y Chile; paz positiva, como el establecimiento de un nuevo entorno de seguridad entre los países señalados $y$, finalmente, elementos de híbridos, especialmente en las relaciones de seguridad entre Colombia y Venezuela o Perú y Chile (Battaglino, 20 I2, pp. I35-I37).

Otorgándole mayor profundidad a aquellos elementos híbridos de seguridad en América Latina, Duarte Villa, Chagas-Bastos y Macedo Braga (2019), enfatizan este camino como un marco alternativo, el cual se concibe como un espacio de tránsito, cambio, superposición y coexistencia de construcción conjunta realizado por diversos agentes (p. 75). En efecto, se infiere que la presencia del estado ha sido indispensable, pero también del multilateralismo y de ciertas prácticas del crimen organizado.

Precisamente, la prevalencia del multilateralismo facilitó históricamente la implementación por parte de los estados de medidas de confianza mutua, instrumentos de resolución pacífica de controversias y canales diplomáticos formales e informales que dan cuenta del arraigo al derecho internacional, a instituciones como la Organización de Estados Americanos (OAE) o acuerdos vinculantes como el Tratado de Asistencia Recíproca (TIAR) de 1947, que crearon una cultura multilateral de primacía de la soberanía y respeto hacia el otro (Herz, Siman y Telles, 20 I7, pp. I23- I48).

Sin embargo, la utilidad y eficacia de los mecanismos descritos, se encuentra actualmente 
bajo profundo cuestionamiento, en la medida que no han logrado anticiparse para solucionar tensiones políticas, económicas o sociales, razón por la cual se estimula la creación y participación estatal en diferentes foros políticos, creando una superposición institucional, que no facilita una mejor efectividad y eficacia, tal como sucedió entre la OEA y la Unión de Repúblicas Suramericanas (UNASUR) durante la primera década del siglo XXI.A pesar que la superposición institucional teóricamente no debiera ser un impedimento para la acción efectiva de la gobernanza regional, las reiteradas disputas respecto a medios y fines para encontrar una mejor solución a los desafíos contemporáneos dejó pasar opciones únicas para asegurar una mediación efectiva (Weiffen, 2017, Pp. 173-198).

Con base en lo anterior, también han surgido discusiones sobre el multilateralismo y su utilidad, pertinencia y capacidad de actuación. En primer lugar, se ha rivalizado ampliamente si la heterogeneidad de los diversos y sobrepuestos foros multilaterales regionales no ha llegado a una especie de cúspide (Malamud y Gardini, 2012, pp. II6-I33), si el multilateralismo ha evolucionado a una forma opcional modular (Gardini, 20I5, pp. 20I-229), en la cual los estados eligen aquello que medianamente satisface sus necesidades e intereses dejando de lado otras disposiciones. En segundo lugar, si los foros regionales responden efectivamente al viejo y tradicional anhelo de mejorar, por un lado, las relaciones entre estados y, por el otro, convertirse en espacios de proyección regional autónomos, diferentes a la imposición de países externos (Deciancio, 2016, pp. I- I4).

Lo anterior ha sido reforzado en el siglo XXI con la creación de foros permanentes, tales como UNASUR, La Alianza Bolivariana para los Pueblos de nuestra América (ALBA) y la Comunidad de Estados Latinoamericanos y Caribeños (CELAC), con lo que se evidencia cierta heterogeneidad ideológica para establecer nuevas identidades colectivas (Gardini y Lambert, 20I I), construyendo una especie de multilateralismo posthegemónico (Riggirozzi y Tussie, 20I2, Pp. I-I6), sobre la cual se observan dos tendencias: por un lado, la intención de acercar a los ciudadanos a la toma de decisiones, diversificar la agenda y los aliados de los países de región y, por el otro, un discurso político que rechaza las instituciones financieras internacionales o el libre mercado sin confrontar, por ejemplo, el exceso de hiperpresidencialismo regional en la toma de decisiones (Legler, 20 I3, pp. 325-352).

En esa misma línea, otros han argumentado que, más que ver a Estados Unidos como un impedimento al fortalecimiento y desarrollo de la gobernanza y el multilateralismo regional, hay que saber extraer los instrumentos idóneos para hacer más efectiva la participación de ese actor en los asuntos regionales, incluidos los temas de seguridad (Petersen y Schulz, 2018, pp. I02-127). En efecto, la heterogeneidad de temas y la participación más activa de instituciones genera un proceso multilateral que debiera estimular una mejor gobernanza, tal como se estableció en las secciones precedentes.

Retomando los factores de gobernanza híbrida de seguridad en la región se destacan en este artículo dos líneas de argumentación, en las que, por un lado, se encuentran los defensores de una visión basada en la geopolítica, el balance de poder y la tensión latente entre estados y, por el otro, una más enfocada en ilustraciones reflectivistas con óptica constructivista, asociativa y cooperativa. 
Nuevamente cobra importancia la reflexión enarbolada tanto por Adler y Greve (2009, Pp. 59-84) como por Duarte Villa, Chagas-Bastos, y Macedo Braga, (2019, pp. pp. 77-100). Los primeros destacan la convivencia de instrumentos propios del realismo como el balance de poder y el bandwagoning, así como el nacimiento paralelo de mecanismos propios de comunidades de seguridad, como las expectativas pacíficas de cambio en fases emergentes o de desarrollo. Los segundos señalan la importancia de prácticas, discursos y el anhelo histórico de consolidar una comunidad imaginada.

En ese sentido, Kacowicz y Mares (2016, pp. I I-30) emplazan la gobernanza regional en términos estratégicos, en los cuales las dinámicas de competencia y conflicto entre estados siguen imperando doscientos años después de independencia.

Los autores, haciendo un recorrido sobre la historia republicana, destacan al menos cinco variables útiles para el análisis de la seguridad: en primer término, las viejas tensiones geopolíticas entre estados determinan situaciones de conflicto y desconfianza; en segundo lugar, subrayan la competencia de liderazgos individuales y personalistas sobre la provisión de bienes comunes y de solución de controversias. En tercer lugar, reconocen que las disputas regionales, han sido avivadas tanto por la competencia interna —es decir entre estados de la región- $y$, al mismo tiempo, por la permisividad frente a la influencia de potencias externas; en cuarto lugar, acentúan la importancia que para los países de la región significa la calidad de la democracia, que facilite los procesos de gobernabilidad interna y, por último, el vínculo entre seguridad y economía en términos de desarrollo económico, esto es, cómo la independencia y autonomía económica, fortalece la seguridad estatal (Kacowics y Mares, 2016, pp. I I-30).

En esa misma línea, otros han profundizado sobre viejas y nuevas tensiones geopolíticas en las que, adicional a enfrentamientos históricos territoriales, se han abierto otras, como la perdida de centralidad de Estados Unidos, la necesaria expansión en los mercados asiáticos y la competencia por el abastecimiento o acceso seguro de recursos naturales estratégicos (Nolte y Wehner, 2016, pp. 33-43). En esencia, los países de la región han encontrado en componentes históricos, estructurales y comportamentales de sus élites, elementos que facilitan o propician la confrontación frene al vecino porque estratégica y políticamente tiene recompensa (Flemes y Wehner, 2015, pp. 163-177).

Lo anterior refleja la dominante huella del realismo político en la que, por un lado, se evidencia el mantenimiento de una zona de paz negativa, por cuanto las rivalidades y tensiones siguen siendo formas de enfrentamiento político e ideológico (Thies, 2016, Pp. II3-126) y, por otro, aunque la guerra no ha sido una forma reiterada para resolver las diputas, las dinámicas del crimen organizado si generan unos niveles de violencia urbana más altos que en otras regiones (Merke, 20II).

Sobre el particular, el carácter híbrido también ha sido explicado por la influencia de dinámicas del crimen organizado. Bandas criminales emergentes en Colombia, y grupos delincuenciales como Primer Comando de la Capital en Brasil desafían el control estatal a partir de instrumentos horizontales y verticales de control sobre la sociedad, los procesos productivos o las dinámicas de delincuencia común, generando una especie de institucionalidad paralela (Duarte 
Villa et al. 2021, pp. 37-39).

En contraposición a lo antes expuesto, se sitúa la línea más interpretativa de la realidad, destacando instrumentos tanto de poder como discursivos y comportamentales, en los cuales sobresalen los aportes de Domínguez, para quien las dinámicas de gobernanza de seguridad, están determinadas por los factores internos que condicionan o refuerzan la actuación internacional de los estados, la resignificación regional a partir de la creación de foros multilaterales alternativos, más allá de la OEA, como la CELAC,UNASUR u ALBA, en los cuales también hay componentes no sólo de comercio e integración, sino también de seguridad y, por último, una cooperación regional limitada con baja densidad institucional, política y presupuestal (Domínguez, 20I7, pp. 53-76).

Adicionalmente, otros señalan que el incipiente desarrollo de comunidades de seguridad debe ser analizado con precisión, subrayando la capacidad real y efectiva de las instituciones internacionales para proveer contornos estables y duraderos de cambio pacífico entre los actores (Weiffen y Duarte Villa, 20 I7, pp. I-26).

De la afirmación anterior se desprenden dos grandes reflexiones. En primer término, las potencias regionales tienen una gran responsabilidad en guiar, construir y proponer espacios de encuentro entre los miembros participantes de los espacios regionales. En este sentido, el papel de Brasil en la construcción de un espacio geopolítico que favoreciera sus intereses (Burges, 2013; Gomes Saraiva, 2010, pp. 577-594), la mayor atracción generada por la ola rosada durante la primera década del siglo XXI, visto como bloque alternativo al predominio estadounidense en la región con un discurso retórico sobre la mayor autonomía regional y la necesidad de abordar temas que impactaran directamente a la población (Belém Lopes y Pimenta de Faria, 2016, Pp. I I53), fueron elementos destacables alrededor de la actividad de las potencias regionales.

En segundo término, la influencia de Estados Unidos sigue siendo un factor determinante, no solo en el ámbito multilateral en el interior de la OEA, a pesar de que esta pierde importancia estratégica (Grabendorff, 20 I8, pp. I53-168) sino también en el relacionamiento económico social y cultural. De hecho, países como Colombia, mantienen una estrecha relación con la potencia del norte y es pieza central en su estrategia de internacionalización (Piñeros Ayala, 20 I 9, pp. 25 I-330).

En efecto, las características de la gobernanza híbrida de seguridad a lo largo del siglo XXI, facilitan observar el carácter contestado del orden imperante a partir de la profunda heterogeneidad retórica y política. Ese hecho, genera al menos tres consecuencias: primero, pone en dificultad los patrones históricos de comportamiento entre estados; segundo, dificulta la efectividad del multilateralismo y la inclusión de otros actores y, finalmente, consolida estructuras fluidas y porosas entre instituciones formales e informales, creadas por grupos al margen de la ley que operan distintas conductas de crimen transnacional organizado (Duarte Villa et al. 202 I, p. 40).

Retomando lo propuesto en el epígrafe primero, tal como lo señalan Sil y Katzenstein (2010), "el eclecticismo analítico es una forma particular de instancia hacia la investigación, en la cual, se aspira a complementar, comprometer, y selectivamente utilizar elementos teóricos para enriquecer y fortalecer las investigaciones académicas" (p. 4l3). 
La gobernanza híbrida en América Latina evidencia de este modo ese eclecticismo entre dos posturas: por un lado, la construcción y consolidación de comunidades de seguridad en las cuales la confianza mutua, la afinidad ideológica y ciertos principios y valores son la guía fundamental y, por el otro, viejas disputas, dinámicas de poder material y desconfianza mutua y reciproca se convierten en el carácter explicativo más destacable (Duarte Villa, 20 I7, pp. pp. 77-100). Con base a lo anterior, la comparación entre comunidades de seguridad y complejos regionales de seguridad cobra más vigencia, en la medida que la primera desenvuelve las medidas de confianza y seguridad colectiva, mientras la segunda se enfoca más en los comportamientos de securitización, competencia y capacidades materiales (Frasson Quenoz y Piñeros Ayala, 20 I4; Duarte Villa, 2017, PP. I3-5I). Como previamente se señaló, ninguna es más fuerte que la otra para determinar con claridad una dinámica de mediano o largo plazo.

Finalmente, el debate sobre gobernanza híbrida no se considera cerrado, por el contrario, se evidencia reactivo frente a patrones de cambio y continuidad, así como a la consolidación de espacios de transición en los que se superponen prácticas, discursos y comportamientos entre actores estatales y no estatales, en ocasiones divergentes y poco claros (Duarte Villa et al. 2019, Pp. 72-94).

\section{Conclusión}

Este artículo ha explorado la gobernanza global en el estudio de las Relaciones Internacionales, y su utilidad para explicar la agenda de seguridad en órdenes regionales, haciendo hincapié en América Latina, señalando tanto su carácter heterogéneo, incompleto y contestado, como los elementos que proveen una superposición entre los estados y las instituciones multilaterales. Se ha destacado igualmente que la gobernanza puede ser más útil al ampliarla a entornos específicos. Retomando el enfoque metodológico, este artículo estimuló la necesidad de construir marcos analíticos y teóricos situados, pragmáticos en el sentido que puedan aprovechar el conocimiento para ser aplicado a casos de estudio eclécticos. En otras palabras, desplegar las herramientas teóricas y conceptuales para la explicación de contornos regionales más allá de los tradicionales euroatlánticos. Sin convertirse en un punto final, se dejan abiertas las puertas para investigaciones más profundas, acerca de las prácticas y los comportamientos regionales entre agentes intervinientes en materia de seguridad.

Los diversos debates en una disciplina centenaria como las Relaciones Internacionales permitieron en palabras de Tickner y Wæver (2009, pp. I-32), ampliar a otras latitudes geográficas las definiciones, prácticas y comportamientos de los agentes que intervienen en temas como seguridad, señalando la relación entre dinámicas globales y efectos locales. Es decir, se abandonan prejuicios y modelos de pensamiento estáticos para reflejar el dinamismo de las cuestiones regionales y su utilidad analítica.

Otros aportes, como los de Adler y Greve (2009, pp. 59-84) o Duarte Villa et. al. (2019, pp. 72-94) facilitaron aplicar la convivencia e hibridez de mecanismos realistas con interpretaciones reflectivistas y mostrar tanto el error de verlas como excluyentes, así como la ventaja de observar los procesos híbridos como medios de transición, en ocasiones sin un destino claro o definido. 
Los elementos políticos e ideológicos seguirán jugando un rol al interior del estado que condiciona sus posturas internacionales. Incluir lo anterior implica romper los estereotipos tradicionales de análisis y situarse en las particularidades propias de la región.

En otras palabras, la gobernanza híbrida de seguridad refleja la apropiación cultural que se le otorga en cada espacio geográfico a las dinámicas y comportamientos específicos que allí se llevan a cabo. Lo anterior permitió observar elementos de renovación, transformación y adaptación, producidos por los cambios políticos, ideológicos o prácticos que constantemente se llevan a cabo por la influencia de los estados, las organizaciones multilaterales o las dinámicas del crimen organizado.

Finalmente, se observó que la tradición continúa teniendo un significado arraigado con lo cual, se ha dado inicio a una mayor contestación - en ocasiones rechazo- del orden particular establecido. En ese orden de ideas, la región atraviesa por una especie de construcción basada en la superposición de órdenes y la convivencia de estructuras híbridas, lo que realza la relevancia de este artículo.

\section{Referencias}

Adler, E.y Barnett, M. (1998). Security Communities. Cambridge University Press.

Adler, E. y Greve, P. (2009). When security community meets balance of power: overlapping mechanisms of security governance. Review of international studies, 59-84.

Barnett, M. y Duvall, R. (2005). Power in global governance. En M. Barnett, y R. Duvall, Power in global governance (pp. I-32). Cambridge University Press.

Battaglino, J. M. (20/2). The coexistence of peace and conflict in south America: toward a new conceptualization of types of peace. Revista brasileira de política internacional, 55 (2), |3|-|5|.

Belém Lopes, D. y Pimenta de Faria, C.A. (2016). When foreign policy meets social demand in Latin America. Contexto internacional, 38 (I), II-53.

Breslin, S. y Croft, S. (2012). Researching regional security governance. En S. Breslin, y S. Croft. Comparative regional security governance (págs. I-22). Routledge.

Burges, S.W. (2013). Brazil as a bridge between old and new powers? International affairs, 89 (3), 577-594.

Buzan, B.,Waever, O.y Wilde, J. D. (1998). Security: a new framework for analysis. Lynne Rienner Publishers.

Buzan, B.y Hansen, L. (2009). The evolution of international security studies. Cambridge University Press.

Caporaso, J.A. y Madeira, M.A. (20I2). Globalization, institutions and governance. Sage Books.

Ceccorulli, M. y Lucarelli, S. (20I4). Security governance: making the concept fit for the analysis of a multipolar, global and regionalized world. European University Institute.

Dabène, O. (2012). Consistency and resilience through cycles of repolitization. En P. Riggirozzi, y D. Tussie. The rise of post-hegemonic regionalism, the case of Latin america (pp. 4I-64). Springer.

Deciancio, M. (2016). International relations from the south: A regional research agenda for global IR. International studies review, I-I4.

Del Arenal, C. (2019). Relaciones Internacionales: Una disciplina liquida. En A. Lozano Vázquez, D. J. Sarquís Ramírez, y D. Jorge. ¿Cien años de relaciones internacionales? (pp. 176-194). Siglo XXI Editores.

Dingwerth, K. y Pattberg, P. (2006). Global Governance as a perspective on world politics. Global governance, 12 (2), 185-203.

Domínguez, R. (2017). Security governance in latin america. En M.A. Suarez, R. DuarteVilla,y B.Weiffen. Power dynamics and regional security in latin america (pp. 53-76). Palgrave.

Domínguez, R. y Velázquez Flores, R. (2018). Global governance. Oxford Research Encyclopedia.

DuarteVilla, R. (2017). Security community of balance of power? Hybrid security governance in Latin America. En M.A. Suarez, R. Duarte Villa, y B.Weiffen. Power dynamics and regional security in latin america (pp. 77-100). Palgrave

Duarte Villa, R. Chagas-Bastos, F. H. y Macedo Braga, C. (2019). Hybrid security governance: an empirical assessment. Latin america politics and society, $6 \mathrm{I}(4), 72-94$.

Duarte Villa, R. Macedo Braga, C.y Ferrerira, M.A. (202I).Violent nonstate actors and the emergence of hybrid governance in south america. Latin american research review, 56 (I), 36-49.

Ellis, E. R. (2016). The impact of China on the security environment of latin america and the caribbean. En D. R. Mares, yA. M. Kacowicz. Routledge handobook of latin american security (pp. 29I-30I). Routledge. 
Fabrinni, S. (2020). The governance of the European Union. En J.-J. Sppon, y N. Ringe.The European Union and beyond: multilevel governance, institutions and beyond (pp. 233-252). ECPR Press.

Flemes, D.y Nolte, D. (20I0). Introduction. En D. Flemes (Ed.). Regional leadership in the global system (pp. I-I 5).Ashgate.

Flemes, D. y Wehner, L. (20I5). Drivers of strategic contestation: the case of south america. International politics, 52(2), 163-177.

Frasson Quenoz, F. y Piñeros Ayala, R. E. (20I4). Complejo regional de seguridad y comunidad de seguridad: algunas consideraciones teóricas. En F. Frasson Quenoz, Seguridad internacional y ordenamientos regionales: Del complejo regional a la comunidad de seguridad (pp. |3-5I). Universidad Externado de Colombia.

Gardini, G. L. (20I5). Towards modular regionalism: the proliferation of Latin American cooperation. Revista brasileira de politica internacional, 20I-229.

Gardini, G. L. y Lambert, P. (20I I). Latin American foreign policy: between ideology and pragmatism. Palgrave.

Gomes Saraiva, M. (20 I0). Brazilian foreign policy towards South America during the Lula administration: caught between South America and Mercosur. Revista brasileira de politica internacional (53), I5 I-I68.

Grabendorff,W. (2018). El impacto de la globalización sobre el peso político del eje transatlántico en el orden internacional. En A. Sebrin. América latina y el caribe frente al nuevo orden internacional (pp. I53-168). Icaria editorial.

Held, D. (20I4). The diffusion of authority. En T. G.Weiss, y R.Wilkinson, International organizations and global governance (pp. 60-72). Palgrave.

Held, D. \& Hale, T. (20I I). Handbook of transnational governance. Polity Press.

Herz, M. (20I4). Regional governance. En T. G.Weiss, y R. Wilkinson. International institution and global governance (pp. 236-250). Routledge.

Herz, M. Siman, M. y Telles, A. C. (2017). Regional organizations, conflict resolution in south america. En M.A. Suarez, R. Duarte Villa, y B.Weiffen. Power dynamics and regional security in Latin America (pp. 123- |48). Palgrave.

Hurrell, A. (2010). Regional powers and the global system from a historical perspective. En D. Flemes. Regional leadership in the gobal system (pp. 15-30). Ashgate.

Jönsson, C. (2018). Theoretical approaches to international organization. Oxford research encyclopedia.

Kacowics, A. M. y Mares, D. R. (2016). Security studies and security in latin america: the first 200 years. En D. R. Mares, y A. M. Kacowics. Routledge handbook of latin america security (pp. I I-30). Routledge.

Kacowicz, A. M. (20I8). Regional governance and global governance: links and explanations. Global governance, 24, 6I79.

Katzenstein, P. y Sil, R. (2008). Eclectic theorizing in the study and practice of international relations. En C. Reus-Smit, y D. Snidal. The oxford handbook of international relations (pp. 109-130). Oxford University Press.

Kirchner, E. J. (20I4). Theoretical debates on regional security governance. European University institute.

Kirchner, E. J., y Domínguez, R. (20l4). Security governance in a comparative regional perspective. European security, 23 (2), I63-178.

Kjær, M. y Kinnerup, K. (2002). Goog governance: how does it relate to human rights? En H.-O.A. Hano. Human Rights and good governance (Pp. I-19). Martinus publishers.

Legler, T. (2013). Post-hegemonic regionalism and sovereignty in latin america: optimists, skeptics, and emerging research agenda. Contexto internacional, 325-352.

Lozano Vázquez, A. (2019). Introducción: a ¿cien años de las Relaciones Internacionales? En A. Lozano Vázquez, D. J. Sarquís Ramírez, y D. Jorge. ¿Cien años de relaciones Internacionales? (pp. 9-27). Siglo XXI Editores.

Luckhurst, J. (2019). La gobernanza global y la complejidad transversal de las relaciones internacionales. En A. Lozano Vázquez, D. J. Sarquís Ramírez, y D. Jorge, ¿Cien años de relaciones internacionales? (pp. I76-194). Siglo XXI Editores.

MacFarlen, S. N. (20I4). Regional organization and global security governance. En T. G. Weiss, y R. Wilkinson. International organization and global governance (pp. 429-44I). Routledge.

Malamud, A. y Gardini, G. L. (2012). Has regionalism peaked? The Latin America quagmire and its lessons. The international spectator, II6-I33.

Marín-Aranguren, E. M.y Trejos-Mateus, D. F. (2020). Del Concepto a la teoría de la gobernanza global: más incluyente con la sociedad civil en las relaciones internacionales. En F. Frasson-Quenoz (Coord.) Pensar, debatir y aportar a las Relaciones Internacionales (pp. 69-I I6). Universidad Externado de Colombia.

Merke, F. (20I I). The primary institutions of the Latin American region interestate society. IDEAS.

Muhr, T. (2019). Geographies of south-south relations and regionalisation process in Latin America-Caribbean. En E. Fiddian-Qasmiyeh, y P. Daley. Routledge handbook of south-south relations (pp. 95-I I I). Routledge.

Murphy, C. N. (20I4). The emergence of global governance. En T. G.Weiss, y R.Wilkinson. International organizations and global governance (pp. 23-35). Routledge.

Nolte, D.y Wehner, L. (2016). Geopolitics in latin america, old and new. En D. R. Mares, y A. M. Kacowicz. Routledge handbook of latin america security (pp. 33-43). Routledge.

Petersen, M.y Schulz, C.-A. (2018). Setting the agenda: a critique of posthegemonic regionalis. Latin america politics and society, $102-127$.

Piñeros Ayala, R. E. (2019). La inserción internacional de Colombia en materia de seguridad y defensa: una cuestión estatal. En G. Palamara (Ed.), Nuevas estrategias de inserción internacional para América Latina (pp. 25I-330). Universidad Externado de Colombia.

Riggirozzi, P. y Tussie, D. (20I2). The rise of post-hegemonic regionalism in Latin America. En P. Riggirozzi, y D. Tussie. 
The rise of post-hegemonic regionalism. The case of Latin America (Pp. I- 16). Springer.

Rocha Valencia, A. y Morales, R. D. (20I I). Potencias medias, potencias regionales. Universidad de Guadalajara.

Rosenau, J. N. (1992). Governance, order and change in world politics. En J. N. Rosenau, y E. O. Czempiel. Governance without government: order and change in world politics (pp. I-29). Cambridge University Press.

Ruggie, J. G. (1993). multilateralism: the anatomy of an institution. En J. G. Ruggie. Multilateralism matters: the theory and praxis of an institutional form (pp. 3-5I). Columbia University Presss.

Sanahuja, J.A. (2017). A Rashmon history. Latin american views and discourses of global governance and multilateralism. En A. Triandafyllidou, Global governance from regional perspective (pp. I8I-208). Oxford University Press.

Sanahuja, J.A. (2019). Ausencias y exclusiones: una mirada reflexiva sobre la constitución de las relaciones internacionales como disciplina. En A. Lozano Vázquez, D. J. Sarquís Ramírez, y D. Jorge. ¿Cien años de relaciones Internacionales? (pp. I32-I53). Siglo XXI Editores.

Serbin, A. (2018). América Latina y el Caribe frente a un nuevo orden mundial: crisis de la globalización, reconfiguración global del poder y respuestas regionales. En A. Serbin. América Latina y el Caribe frente a un nuevo orden internacional (Pp. 13-35). Icaria Editores.

Sil, R. y Katzenstein, P.J. (20I0). Analytic eclecticism in the study of world politics: reconfiguring problems and mechanism across research traditions. Perspective on politics, 8(2), 4I I-43 I.

Thakur, R. y Van Langenhove, L. (2006). Enhancing global governance through regional integration. Global governance, I2, 233-240.

Thies, C. G. (2016). Traditional security. War and peace. En D. R. Mares, y A. M. Kacowicz. Routledge handobook of latin american security (pp. I13-126). Routledge.

Tickner, A. B. (2003). Hearing latin american voices in international relations theory. International studies perspectives (4), 325-350.

Tickner, A. B. y Wæver, O. (2009). Introduction geocultural epistemologies. En A. B. Tickner, y O. Wæver. International relations, scholarship around the world. Worlding beyond the west (Pp. I-32). Routledge.

Triandafyllidou, A. (2017). Global governance from regional perspective. En A. Triandafyllidou. Global governance from critical perspective (pp. 3-24). Oxford University Press.

UN. (1995). Our global neighbourdhood: the report of the commission on global governance. United Nations.

Weiffen, B. (2017). Institutional overlap and responses to political crisis in South America. En M.A. Suarez, R. Duarte Villa, y B.Weiffen, Power dynamics and regional security in latin america (pp. 173-198). Palgrave.

Weiffen, B., y DuarteVilla, R. (2017). Re-thinking latin america regional security: the impact of power and politics. En M. A. Suarez, R. Duarte Villa, y B.Weiffen. Power dynamics and regional security in latin america (Pp. I-26). Palgrave.

Weiss, T. G. y Wilkinson, R. (20l4). International organization and global governance. what matters and why. En T. G. Weiss, y R.Wilkinson. International organization and global governance (pp. 3-19). Routledge.

Zürn, M. (20।8). A theory of global governance. Oxford University Press. 


\section{RELACIONES INTERNACIONALES}

Revista académica cuatrimestral de publicación electrónica Grupo de Estudios de Relaciones Internacionales (GERI)

Universidad Autónoma de Madrid, España

https://revistas.uam.es/relacionesinternacionales

ISSN 1699 - 3950

f facebook.com/RelacionesInternacionales

3. twitter.com/RRInternacional

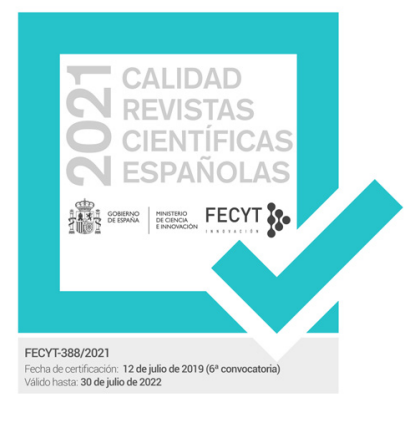

